\title{
Catálogo de Benefícios Relatados por Organizações que Implementaram Melhoria de Processos de Software
}

\author{
Diego Cruz e Gleison Santos
}

Programa de Pós-Graduação em Informática - Universidade Federal do Estado do Rio de Janeiro (UNIRIO) - Av. Pasteur, 458, Urca, CEP 22290-240 - Rio de Janeiro, RJ

\{diego.dacruz, gleison.santos\}@uniriotec.br

\begin{abstract}
Awareness of software process improvement (SPI) benefits may motivate organisations to decide to adopt and maintain practices in maturity models such as MRMPS-SW and CMMI-Dev. We performed a systematic data collection and analysis to identify and catalog informations about SPI benefits obtained in the state of practice. We conducted a systematic mapping review and an expert panel that were analyzed by means of a thematic analysis with Grounded Theory procedures. After analyzing 57 experience reports and 4 experts interviews, we identified 121 benefit sheets that were organized in a SPI benefit catalog. The experts evaluation generated evidences that the sheets' structure is proper and relevant to the organisational context. We also found that its content can positively influence motivation of team affected by the SPI initiative besides the high management.
\end{abstract}

Resumo. A percepção dos benefícios gerados por implementação de melhoria de processos de software (SPI) é um fator motivacional para que organizações decidam adotar e manter práticas propostas em modelos de maturidade como MR-MPS-SW e CMMI-Dev. Com objetivo de contribuir para maior evidência dos possíveis benefícios da adoção de práticas propostas por modelos e normas internacionais de SPI, esta pesquisa englobou uma abordagem sistemática de coleta e análise de dados para identificar e catalogar informações sobre benefícios de SPI obtidos no estado da prática. O método contempla execução de um mapeamento sistemático da literatura e Expert Panel cujos resultados foram analisados utilizando procedimentos de análise temática com codificação baseada em Grounded Theory $(G T)$. A análise dos 57 relatos de experiência de implementação de SPI selecionados e das 4 entrevistas com especialistas em SPI resultaram na identificação de 121 fichas de benefícios de SPI que foram organizadas em um catálogo de benefícios. A avaliação por especialistas mostrou indícios de que a estrutura é adequada e relevante ao contexto de SPI e de que o conteúdo poderia influenciar positivamente na motivação de equipe envolvida na SPI e da alta gestão.

\section{Introdução}

O sucesso das organizações desenvolvedoras de software cada vez mais depende da qualidade final dos seus produtos em equilíbrio com o cumprimento dos prazos, custos e escopo estabelecidos, assim como, a minimização das incertezas e maximização das oportunidades que cercam um projeto, tudo isso em um menor tempo possível de desenvolvimento [Neto et al. 2013]. Para guiar as organizações no aumento da maturidade organizacional, bem como o aumento da sua capacidade de desenvolver softwares, foram criados padrões internacionais como a ISO/IEC 12207 [ISO/IEC 2008], 
ISO/IEC 15504 [ISO/IEC 2003] e modelos de qualidade de processo de software como o CMMI-DEV (Capability Maturity Model Integration for Development) [CMMI Product Team 2010] e o MR-MPS-SW (Modelo de Referência MPS para Melhoria de Processos de Software) [SOFTEX 2016]. A adoção de práticas propostas por modelos e normas internacionais de melhoria de processos de software (SPI, do inglês Software Process Improvement) é um processo social cujos fatores de sucesso são amplamente discutidos na literatura. Problemas como a falta de apoio e comprometimento alta direção com as práticas de melhoria, bem como dos colaboradores à execução de atividades propostas pelos modelos pode gerar o fracasso ou descontinuação das iniciativas em organizações. Nesse contexto, autores como Montoni e Rocha (2011) acreditam que a visualização dos possíveis benefícios de SPI pelos colaboradores pode contribuir para redução da resistência a mudanças durante a introdução de práticas propostas por modelos e normas internacionais de SPI. Coleman e O'Connor (2008) e Travassos e Kalinowski (2009) acreditam que evidenciar os possíveis benefícios da SPI promove o apoio da alta gestão, garantindo a manutenção das atividades de melhoria de processos na organização.

Este trabalho tem por objetivo contribuir para a maior visibilidade sobre os possíveis benefícios de melhoria de processos de software por meio da elaboração de um catálogo com benefícios relatados por organizações que implementaram melhoria de processos de software baseada em modelos de maturidade, como o MR-MPS-SW [SOFTEX 2016] e o CMMI-DEV [CMMI Product Team 2010] e em normas internacionais, como a ISO/IEC 15504 [ISO/IEC 2003]. A metodologia de pesquisa inclui a coleta, análise de dados e avaliação de artefatos baseada nas diretrizes da Design Science Research [Dresh et al. 2015]. A próxima seção apresenta uma revisão da literatura, a seção 3 apresenta o planejamento, execução e principais resultados do estudo, suas limitações e ameaças à validade e na seção 4 é apresentada a conclusão.

\section{Benefícios de Melhoria de Processos de Software}

A aplicação de boas práticas de Engenharia de Software pode melhorar o desempenho das organizações com respeito a custo, prazo, produtividade, qualidade, satisfação do cliente e retorno do investimento e, consequentemente, aumentar sua vantagem competitiva [Santos 2011]. A melhoria de processos de software (SPI) tem como objetivo compreender o processo de software, como ele é usado dentro de uma organização e, assim, conduzir a implementação de mudanças para que esse processo alcance metas específicas, tais como obter maior qualidade dos produtos ou reduzir custos [Coleman et al. 2008]. Assim, organizações têm adotado normas e modelos de melhoria de processo como referência para obter a melhoria desejada em seus programas e projetos, visando alcançar maior maturidade no desenvolvimento de software e melhor desempenho no negócio [Cerdeiral 2008].

Dentre as normas e modelos de qualidade mais comuns estão as normas ISO/IEC 12207 [ISO/IEC 2008], ISO/IEC 15504 [ISO/IEC 2003] e os modelos de maturidade CMMI-DEV [CMMI Product Team 2010] e o MR-MPS-SW [SOFTEX 2016].

Decisões por adotar novas práticas para melhoria de processos organizacionais devem estar alinhadas aos objetivos de negócio, assim, é fundamental que setores estratégicos e tomadores de decisão estimem o Retorno sobre Investimento (do inglês 
Return On Investment ou ROI), que consiste na razão entre a representação financeira dos benefícios e a representação financeira do custo. Na SPI, é importante que os benefícios não só excedam os custos, mas os ultrapassem suficientemente para justificar as dificuldades associadas à adoção das práticas [Rico, 2002], pois as iniciativas de melhoria necessitam de um investimento significante e com benefícios muitas vezes invisíveis, o que pode levar uma relação custo-benefício fora do esperado pelas organizações [Solingen 2004]. Para analisar o impacto das iniciativas de SPI, organizações frequentemente utilizam a estratégia de comparação pré-pós, ou seja, analisar o contexto antes e depois da implementação da SPI para verificar se os objetivos de melhoria foram alcançados [Unterkalmsteiner et al. 2012]. Nesse contexto, é essencial que organizações tenham ciência dos possíveis ganhos proporcionados pela SPI para definirem de objetivos iniciais de implementação realistas e evitar frustrações levariam ao abandono das práticas de melhoria.

Em uma abordagem que sugere a comparação pré-pós, Ramos et al. (2013) definiram uma estratégia para análise do Retorno sobre o Investimento com base na expectativa da organização. A estratégia consiste em identificar benefícios esperados pela organização com base nos objetivos de negócio e identificar as medidas necessárias para avaliar o alcance dos objetivos após a institucionalização do processo. Mcloughlin (2010) também define uma estratégia para implementação de SPI orientada a objetivos, denominada Rosetta Stone Metodology, baseada no paradigma Goal-Question-Metric (GQM) [Basili 1994]. Em sua pesquisa, o autor realiza um mapeamento entre as áreas de processos do CMMI e uma árvore de benefícios definida por Goyal et al. [2001 apud Mcloughlin 2010] para permitir que se identifique as áreas de processos do CMMI a serem implementadas com base nos objetivos organizacionais. A partir dos trabalhos de Ramos et al. (2013) e Mcloughlin (2010) foi identificada uma lista considerável de benefícios e medidas que serviram para apoiar suas abordagens e facilitar organizações na definição de metas iniciais de implementação. Apesar disso, a árvore de benefícios usada por Mcloughlin (2010) não é específica de SPI e não há detalhes que propiciem o entendimento mais significativo dos benefícios. Não é possível saber, por exemplo, "que fatores podem influenciar na produtividade?", “em que contexto de SPI o aumento da produtividade foi relatado?" ou "que outros benefícios são gerados pelo aumento da produtividade?".

Todavia, considera-se que a exploração e coleta de detalhes como os referentes a essas questões possam auxiliar a compreensão e reconhecimento dos benefícios da SPI por profissionais e gestores de organizações inexperientes em SPI e de organizações engajadas em iniciativas de melhoria de processos de software. Nesse sentido, as próximas seções apresentam as etapas que levaram à identificação de benefícios associados a iniciativas de SPI baseadas em modelos de maturidade e normas internacionais.

\section{Planejamento e execução do Estudo}

A Design Science Research (DSR) é um método que fundamenta e operacionaliza a condução de pesquisas quando o objetivo a ser alcançado é um artefato ou uma prescrição. O planejamento desse estudo baseou-se em [Dresh et al.2015] que define duas 
etapas principais para pesquisas em DSR: (i) Projeto e desenvolvimento do artefato e (ii) Avaliação do artefato. A etapa de projeto e desenvolvimento do artefato desse estudo compreende a coleta de dados por meio de mapeamento sistemático da literatura (MSL) e análise qualitativa utilizando procedimentos de Grounded Theory (GT), seguido de auditoria dos procedimentos de codificação e definição da estruturação do catálogo. Para a etapa de avaliação do artefato, planejou-se um painel de especialistas para operar tanto como meio de avaliação do catálogo, como também uma fonte secundária para coleta de dados relativos ao objeto de pesquisa (benefícios). A Figura 1 apresenta os elementos envolvidos na criação do artefato proposto nesse estudo, enquanto a Tabela 1 apresenta as atividades envolvidas.

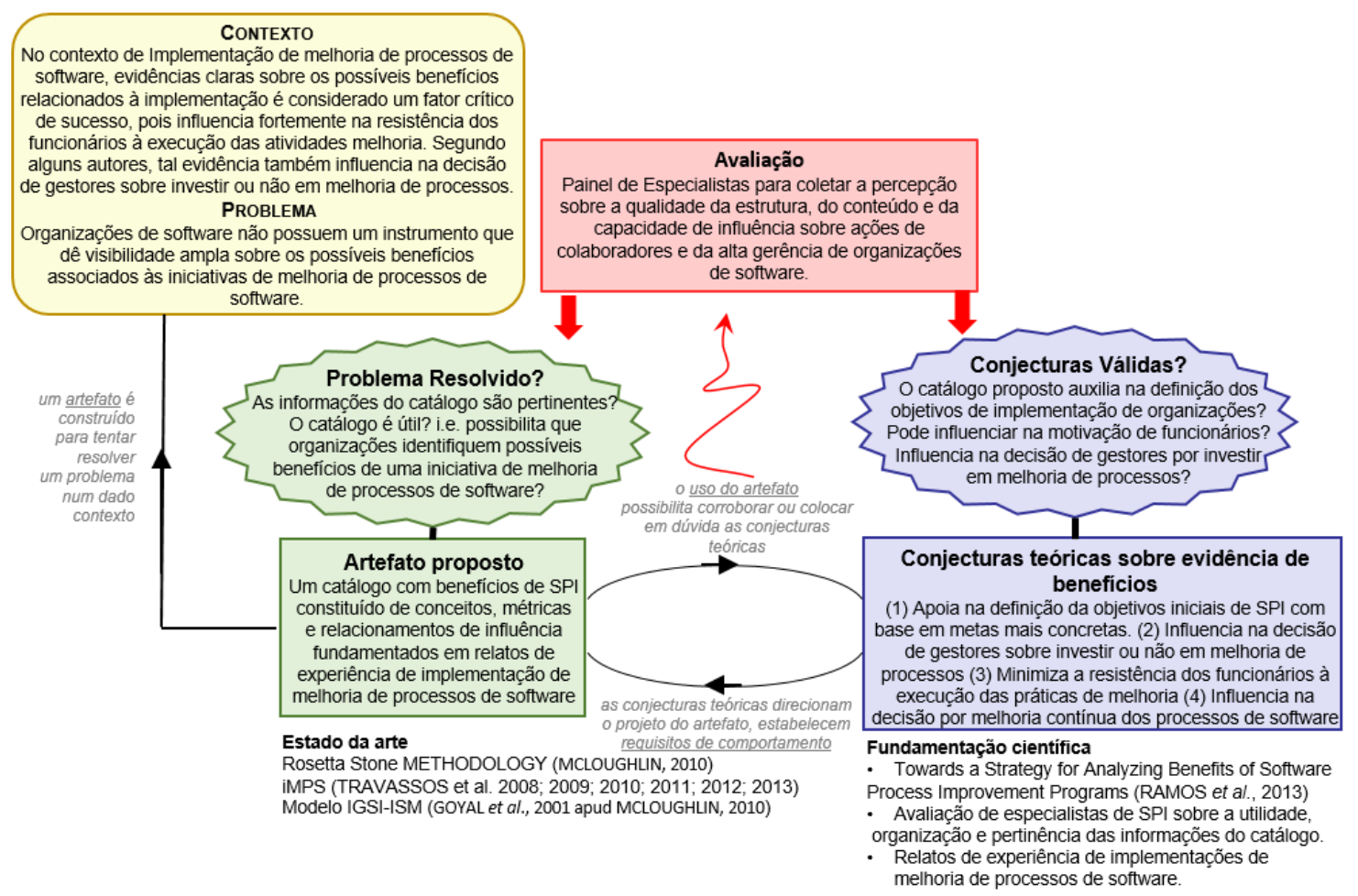

Figura 1 - Modelo de pesquisa em Design Science Research

Tabela 1 - Atividades da metodologia de pesquisa baseada em Design Science Research

Projeto e desenvolvimento do artefato

\begin{tabular}{|c|c|c|c|}
$\begin{array}{c}\text { Mapeamento Sistemático da } \\
\text { Literatura (MSL) } \\
\begin{array}{c}\text { Seleção de publicações no } \\
\text { escopo de pesquisa }\end{array}\end{array}$ & $\begin{array}{c}\text { Análise qualitativa } \\
\text { Análise temática das } \\
\text { publicações selecionadas no } \\
\text { MSL, utilizando } \\
\text { procedimentos de GT }\end{array}$ & $\begin{array}{c}\text { Auditoria por pares } \\
\text { Auditores revisam os códigos } \\
\text { gerados durante o } \\
\text { procedimento de codificação }\end{array}$ & $\begin{array}{c}\text { Definição do artefato } \\
\text { A estrutura do catálogo é } \\
\text { definida e as informações são } \\
\text { organizadas em um layout } \\
\text { estabelecido }\end{array}$ \\
\hline Avaliação do artefato & \multicolumn{3}{|c|}{} \\
\hline $\begin{array}{c}\text { Painel de especialistas } \\
\text { Entrevistas com especialistas } \\
\text { para avaliar a forma e } \\
\text { conteúdo do catálogo de } \\
\text { benefícios }\end{array}$ & $\begin{array}{c}\text { Análise qualitativa } \\
\text { Análise temática das } \\
\text { entrevistas utilizando } \\
\text { procedimentos de GT }\end{array}$ & $\begin{array}{c}\text { Auditoria dos novos } \\
\text { achados } \\
\text { Os novos achados são } \\
\text { auditados por um auditor } \\
\text { experiente em SPI }\end{array}$ & $\begin{array}{c}\text { Atualização do catálogo } \\
\text { Os novos achados são } \\
\text { mesclados aos obtidos no } \\
\text { MSL }\end{array}$ \\
\hline
\end{tabular}

\subsection{Protocolo de Mapeamento Sistemático}

O protocolo do mapeamento sistemático da literatura foi desenvolvido com base nas 
diretrizes propostas por Petersen et al. (2015) que delimitam quatro etapas essenciais a serem seguidas: (i) definição de questões de pesquisa ou escopo do mapeamento; (ii) realização da pesquisa de estudos primários relevantes; (iii) extração de dados; e (iv) análise de síntese dos dados.

O MSL proposto nesse estudo visa identificar estudos primários caracterizados como relatos de experiência de implementação de modelo ou norma de melhoria de processos de software e que relatem benefícios obtidos pela adoção de práticas de SPI no contexto da indústria de software nacional e internacional, com objetivo de identificar benefícios de SPI e informações sobre fatores que influenciam positivamente na ocorrência, fatores que influenciam negativamente na ocorrência, contexto de melhoria de processos em que foram relatados, a relação com outros benefícios e as medidas ou técnicas utilizadas para acompanhamento de ocorrência. O protocolo de MSL completo encontra-se disponível em [Cruz, 2016].

Questões de pesquisa: foi formulada a seguinte questão principal de pesquisa: $Q P 1$. Quais benefícios são relatados por organizações que implementaram melhoria de processos de software? Foram também formuladas as seguintes questões secundárias: QS 1. Quais fatores exercem influência positiva na obtenção dos benefícios relatados?; QS 2. Que métodos a organização utiliza para constatar a ocorrência dos benefícios relatados?; QS 3. Em que contexto de melhoria de processos de software os benefícios ocorrem?; QS 4. Em que publicações os benefícios foram relatados?; QS 5. Qual relação entre os benefícios relatados?

Escopo de pesquisa: A pesquisa se deu a partir de consulta estruturada nas bibliotecas digitais da Scopus e Compendex, pela robustez e abrangência de indexação (que considera boa parte também das publicações da ACM Digital Library e IEEE Xplore) [Ribeiro e Travassos 2016], e a partir de consultas manuais nos anais do Workshop Anual de Melhoria de Processos de Software (WAMPS) e do Simpósio Brasileiro de Qualidade de Software (SBQS), por serem os maiores eventos específicos em Qualidade de Software no Brasil, com grande volume de publicações relacionadas a SPI.

Para a Scopus não houve restrições de data. Para a Compendex, o estudo englobou o período compreendido entre 1989 (ano de lançamento do CMM) e 2016. Nestas duas fontes, foi utilizada uma expressão de busca. Todos os anais do SBQS entre 2002 e 2015 e do WAMPS entre 2005 e 2015 foram considerados. Foram aceitos trabalhos em inglês e português. Para estes dois eventos foi feito uma busca manual em todos os trabalhos.

Métodos de busca de publicações: As etapas do mapeamento sistemático incluíram: (i) Seleção e catalogação preliminar das publicações; (ii) Leitura dos títulos e abstracts com aplicação dos critérios de inclusão e exclusão para identificar publicações em potencial para a pesquisa. Em caso de dúvida, a publicação foi incluída no escopo para leitura completa; (iii) Aplicação de critérios de inclusão e exclusão com base na leitura completa das publicações selecionadas na etapa de leitura dos títulos e abstracts; (iv) Análise qualitativa sobre publicações incluídas no escopo de pesquisa utilizando conceitos de Grounded Theory; e (v) Auditoria dos procedimentos de codificação. 
Considerou-se os seguintes critérios de inclusão (CI) e exclusão (CE): CII Publicações que relatem uma ou mais experiências de implementação de melhoria de processos de software, caracterizando relatos de experiência; CI2 - Artigos que relatem benefícios da implementação de SPI (* apenas durante leitura completa); CE1 - O artigo não está relacionado ao contexto de Engenharia de Software; CE2 - O artigo está escrito em idioma diferente do inglês e português $C E 3$ - Não foi possível ter acesso ao trabalho completo; CE4 - É uma apresentação de conferência.

Definição de expressões de busca: A elaboração da expressão de busca partiu da composição: (benefit or benefits) AND (SPI OR 'software process improvement') com o objetivo de identificar novas palavras-chave. Com base nas publicações que retornaram na primeira execução, foram identificadas palavras frequentemente citadas em títulos e resumos de publicações caracterizadas como relatos de experiência. Assim, a segunda versão da expressão de busca compreendeu os termos: ("software process improvement" OR spi OR cmmi OR cmm OR "maturity level" OR "mps.br") AND (benefit OR tangible OR intangible OR objective OR goal OR "success factors" OR csf OR motivator OR motivation $O R$ lesson $O R$ "experience report" OR roi OR investment). Esta expressão foi executada no engenho de busca Scopus e, após análise dos resultados foi revista e identificaram-se termos que ampliavam consideravelmente o número de falso-positivos. As palavras-chave removidas da expressão foram: objective, csf, motivator e motivation. A decisão por remoção destes termos foi baseada nos artigos já selecionados e em execuções-teste para avaliar a possível perda, onde concluiu-se que não haveria perda significativa na execução da expressão atualizada na base da Compendex.

Resultados da execução do protocolo: Foram identificados 57 relatos de experiência. A Tabela 2 sumariza a seleção de publicações nos engenhos de busca e anais analisados.

Tabela 2- Seleção de publicações nos engenhos de busca automatizada e anais de eventos

\begin{tabular}{|l|c|c|c|c|}
\hline \multirow{2}{*}{\multicolumn{1}{|c|}{ Totais }} & \multicolumn{4}{c|}{ Fontes } \\
\cline { 2 - 5 } & SCOPUS & COMPENDEX & SBQS & WAMPS \\
\hline Total de artigos analisados & 1033 & 347 & 388 & 217 \\
\hline Artigos no escopo & 22 & 4 & 11 & 20 \\
\hline Total de artigos no escopo & \multicolumn{4}{|c|}{57} \\
\hline
\end{tabular}

Os relatos de implementação de SPI que entraram no escopo de pesquisa são de organizações de diversos países, principalmente no Brasil e Estados Unidos. Das organizações estudadas, 35 são brasileiras ou multinacionais que implementaram SPI na sede localizada no Brasil, 8 organizações dos Estados Unidos, 2 da Irlanda e 1 de cada um dos seguintes países: Canadá, Argentina, Turquia, Áustria, Índia, Alemanha e China. Uma organização implementou em duas sedes, sendo uma na Alemanha e outra na Holanda. Em 4 publicações não foi possível identificar o país da iniciativa.

\subsection{Análise Qualitativa dos Benefícios de Melhoria de Processos de Software}

Para responder às questões de pesquisa, uma análise temática foi realizada utilizando conceitos de codificação aberta (open coding) e codificação axial (axial coding) da Grounded Theory $(G T)$ ou Teoria fundamentada nos dados [Strauss e Corbin, 2008]. As análises foram realizadas com apoio da ferramenta ATLAS.ti, que é específica para 
análise qualitativa desta natureza (disponível em http://www.atlasti.com).

Na pré-análise de relatos de experiência foi realizada a atividade da análise temática conhecida como "leitura flutuante" [Bardin 1977] que objetivou gerar impressões iniciais acerca do material a ser analisado e onde foi possível identificar as principais categorias e subcategorias que poderiam ser exploradas no estudo. No decorrer da análise, as categorias foram tornando-se mais evidentes até que foi possível definir o modelo de análise apresentado na Figura 2 que representa o esquema teórico utilizado para codificação e define relacionamentos entre as classes de dados identificadas nesse estudo. A classe central da Figura 2 (Benefícios de Melhoria de Processos de Software) é referente à questão principal de pesquisa, enquanto seus atributos e atributos aninhados (subatributos) fazem referência às questões de pesquisa secundárias. Já as duas classes superiores (Categoria de Benefícios de Melhoria de Processos de Software e Grupo de Benefícios de Melhoria de processos de software) correspondem a agrupamentos realizados durante as etapas de codificação axial.

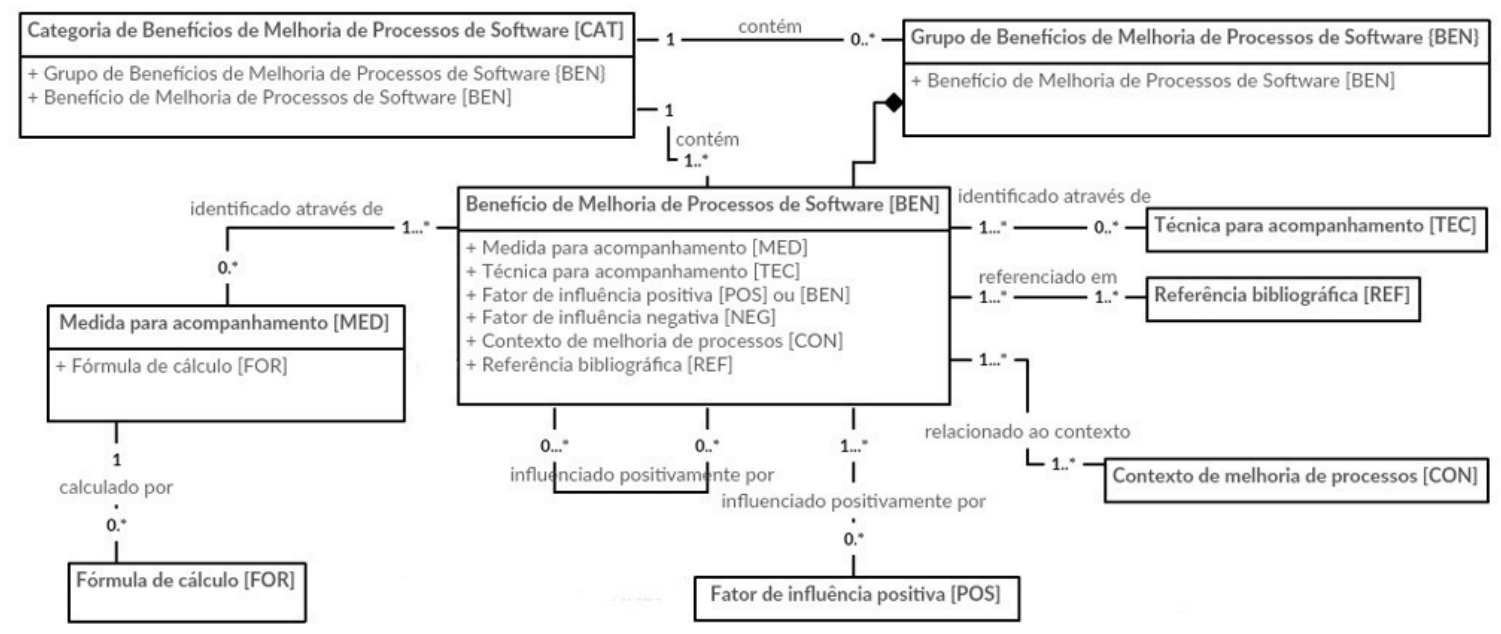

Figura 2: Modelo para análise dos benefícios de melhoria de processos de software

Benefícios com definições similares foram associados a uma descrição mais abstrata que na Figura 2 é representada pela classe Grupo de Benefícios de Melhoria de Processos de Software. O grafo da Figura 3 exemplifica a formação de um Grupo de Benefícios de Melhoria de processos de software. Os números entre chaves no fim dos nomes dos nós representam respectivamente o grau (quantidade de trechos de artigo que estão associados ao nó) e a densidade (quantidade de elementos ligados ao nó). Nesse contexto, o Grupo \{BEN002\} é uma representação abstrata dos benefícios [BEN059], [BEN174] e [BEN023] de modo que um conjunto de benefícios é definido em um único código, denominado grupo de benefícios, que, portanto, herda todos os relacionamentos dos benefícios que o compõem, como medidas e fatores de influência positiva e negativa.

Finalizada a primeira etapa de codificação, foram identificados 169 Benefícios de SPI, 119 Fatores de influência positiva, 3 fatores de influência negativa, 35 Medidas para acompanhamento, 17 Fórmulas, 13 Técnicas para acompanhamento, 57 Referências bibliográficas e 29 contextos de ocorrência. Na segunda fase, benefícios semelhantes foram agrupados reduzindo de 169 para 121 benefícios que, por fim, foram distribuídos 
em 12 categorias de benefício.

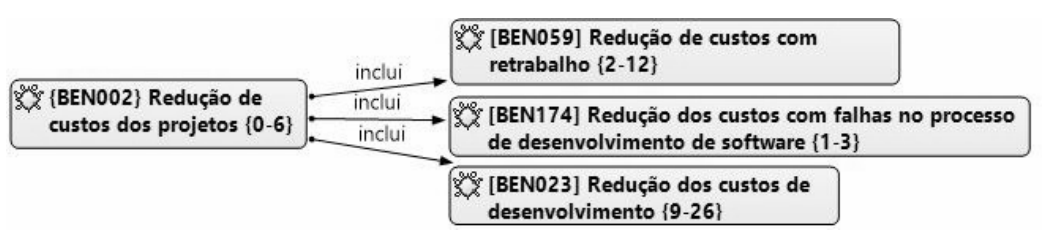

Figura 3 - Exemplo de agrupamento de benefícios

A Tabela 3 apresenta as 12 categorias de benefícios criadas durante a análise qualitativa, a quantidade de benefícios e/ou grupos de benefícios existentes em cada categoria e exemplos de benefícios e/ou grupos de benefício contidos nas categorias.

Tabela 3 - Categorias de benefícios identificadas na codificação axial

\begin{tabular}{|c|c|c|}
\hline Categoria de benefícios & $\begin{array}{c}\text { Benefí- } \\
\text { cios }\end{array}$ & Exemplos de benefícios/grupos de benefícios \\
\hline $\begin{array}{l}\text { CAT001 - Benefícios relacionados } \\
\text { a Ganhos Financeiros e redução de } \\
\text { custo operacional }\end{array}$ & 6 & $\begin{array}{l}\text { Aumento da receita/lucro, Aumento do faturamento, Maior } \\
\text { visibilidade dos lucros, Redução de custos dos projetos e } \\
\text { Retorno do investimento (Ganhos Financeiros). }\end{array}$ \\
\hline \begin{tabular}{|l|} 
CAT002 - Benefícios Relacionados \\
à Melhoria da Comunicação e \\
Relacionamento na Organização
\end{tabular} & 5 & $\begin{array}{l}\text { Melhor comunicação entre colaboradores e equipes/projetos, } \\
\text { Melhor definiçãa dos canais de comunicação e Melhor } \\
\text { comunicação entre o departamento de desenvolvimento, a alta } \\
\text { direção e representantes das organizações clientes. }\end{array}$ \\
\hline \begin{tabular}{|l|} 
CAT003 - Benefícios Relacionados \\
ao Ambiente de Trabalho
\end{tabular} & 11 & $\begin{array}{l}\text { Aumento da satisfação dos colaboradores, Redução na } \\
\text { sobrecarga de trabalho e Aumento da moral dos colaboradores. }\end{array}$ \\
\hline \begin{tabular}{|l|} 
CAT004 - Benefícios Relacionados \\
à Mudança Cultural na \\
Organização
\end{tabular} & 11 & $\begin{array}{l}\text { Maior comprometimento de envolvidos no projetos e Maior } \\
\text { atenção da equipe de projetos com a qualidade dos } \\
\text { produtos de trabalho }\end{array}$ \\
\hline $\begin{array}{l}\text { CAT005 - Benefícios Relacionados } \\
\text { à Qualidade de Relacionamento } \\
\text { com o Cliente }\end{array}$ & 7 & $\begin{array}{l}\text { Maior participação/envolvimento dos clientes nos projetos, } \\
\text { Mais liberdade para os clientes solicitarem mudanças e } \\
\text { Redução de problemas com clientes. }\end{array}$ \\
\hline $\begin{array}{l}\text { CAT006 - Benefícios Relacionados } \\
\text { ao Planejamento de Projetos }\end{array}$ & 7 & $\begin{array}{l}\text { Maior precisão na elaboração de estimativas, Maior } \\
\text { previsibilidade da qualidade do produto e Maior capacidade de } \\
\text { negociação de mudanças com os clientes. }\end{array}$ \\
\hline $\begin{array}{l}\text { CAT007 - Benefícios Relacionados } \\
\text { à Qualidade de Gerenciamento de } \\
\text { Projetos }\end{array}$ & 11 & $\begin{array}{l}\text { Melhor priorização de projetos de desenvolvimento de } \\
\text { software, Maior controle sobre os projetos e Melhor } \\
\text { visibilidade da distribuição de tarefas e evolução do produto. }\end{array}$ \\
\hline $\begin{array}{l}\text { CAT008 - Benefícios Relacionados } \\
\text { à Qualidade de Execução de } \\
\text { Projetos }\end{array}$ & 29 & $\begin{array}{l}\text { Redução do tempo necessário para realização de tarefas, } \\
\text { Redução de retrabalho, Identificação de erros nas fases iniciais } \\
\text { do projeto e Aumento da produtividade. }\end{array}$ \\
\hline \begin{tabular}{|l|} 
CAT009 - Benefícios Relacionados \\
à Organização e Alta Direção
\end{tabular} & 13 & $\begin{array}{l}\text { Aumento da satisfação da alta gerência, Melhoria do time-to- } \\
\text { market e Maior e melhor continuidade dos serviços. }\end{array}$ \\
\hline \begin{tabular}{|l|} 
CAT010 - Benefícios Relacionados \\
à Qualidade dos Produtos de \\
Trabalho
\end{tabular} & 4 & $\begin{array}{l}\text { Aumento da qualidade do produto / Redução da } \\
\text { densidade de defeitos, Maior padronização dos processos e } \\
\text { produtos de trabalho e Melhoria da qualidade dos requisitos. }\end{array}$ \\
\hline $\begin{array}{l}\text { CAT011 - Benefícios Relacionados } \\
\text { à Atração, Retenção e Capacitação } \\
\text { de Profissionais de Software }\end{array}$ & 7 & $\begin{array}{l}\text { Maior número de profissionais interessados em trabalhar para } \\
\text { a organização, Redução da rotatividade de profissionais e } \\
\text { Maior facilidade para treinamento de colaboradores. }\end{array}$ \\
\hline \begin{tabular}{|l|} 
CAT012 - Benefícios Relacionados \\
ao Programa de Melhoria de \\
Processos de Software
\end{tabular} & 10 & $\begin{array}{l}\text { Institucionalização dos processos de software e Geração de } \\
\text { informações sobre os resultados da melhoria de processos de } \\
\text { software que auxiliam gerentes na tomada de decisão }\end{array}$ \\
\hline
\end{tabular}


Com relação à questão de pesquisa principal QP1 "Quais benefícios são relatados por organizações que implementaram melhoria de processos de software?", 169 benefícios foram identificados, dos quais os mais citados em publicações distintas são apresentados na Tabela 4 .

Tabela 4 - Benefícios mais citados

\begin{tabular}{|l|c|}
\hline Nome do Benefício & Citações \\
\hline BEN028 - Aumento da qualidade do produto & 20 \\
\hline BEN004 - Melhor estimativa de prazo & 17 \\
\hline BEN031 - Aumento da produtividade & 17 \\
\hline BEN025 - Redução da taxa de defeitos & 16 \\
\hline BEN029 - Aumento da satisfação do cliente & 12 \\
\hline BEN003 - Redução de retrabalho & 11 \\
\hline BEN023 - Redução dos custos de desenvolvimento & 9 \\
\hline BEN030 - Identificação de erros nas fases iniciais do projeto & 7 \\
\hline BEN065 - Aumento da satisfação dos colaboradores & 7 \\
\hline BEN145 - Redução do número de defeitos encontrados durante a avaliação do cliente & 7 \\
\hline
\end{tabular}

Com relação à questão secundária QS1 "Quais fatores exercem influência positiva na obtenção dos benefícios relatados?", foram identificados 119 fatores de influência positiva, ou seja, fatores que podem facilitar a ocorrência dos benefícios aos quais estão relacionados. O grafo da Figura 4 apresenta exemplos de fatores que influenciam positivamente no benefício [BEN004] - "Melhor estimativa de prazo".

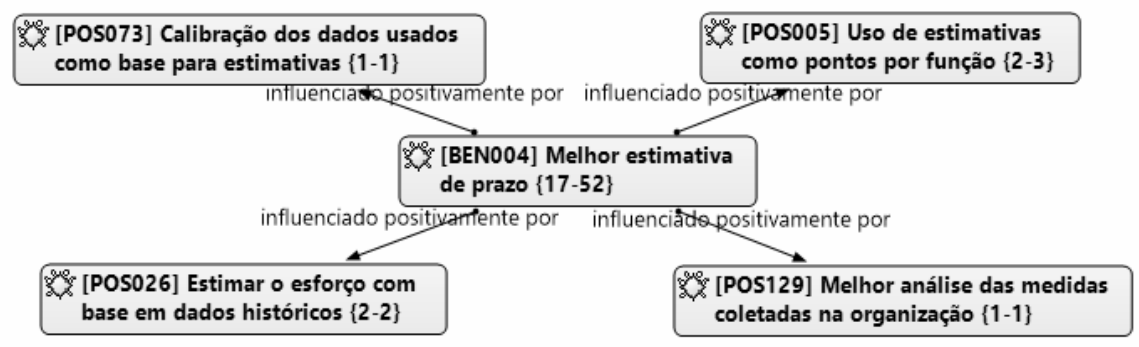

Figura 4 - Exemplo da codificação de fatores de influência positiva

Com relação à questão secundária QS2 - “Que métodos a organização utiliza para constatar a ocorrência dos benefícios relatados?", foram identificadas 35 medidas para acompanhamento, 17 Fórmulas e 13 Técnicas para acompanhamento. As medidas mais citadas pelos autores estão representadas no gráfico da Figura 5. Para a maior parte das medidas identificadas não foi possível identificar a fórmula nas publicações analisadas, entretanto, 11 das 35 medidas são diretas, ou seja, não necessitam de fórmulas, por exemplo "Quantidade de defeitos no produto final". Das 24 medidas que não são diretas, 15 tiveram fórmulas definidas por algum autor. Já as "técnicas para acompanhamento" são maneiras de se identificar a ocorrência de benefícios que muitas vezes não são mensurados numericamente e a coleta de dados é realizada a partir da percepção das pessoas envolvidas. Nesse estudo, a única técnica que obteve mais de uma citação foi a "[TEC001] Elaborar um questionário de satisfação", com 5 citações. 


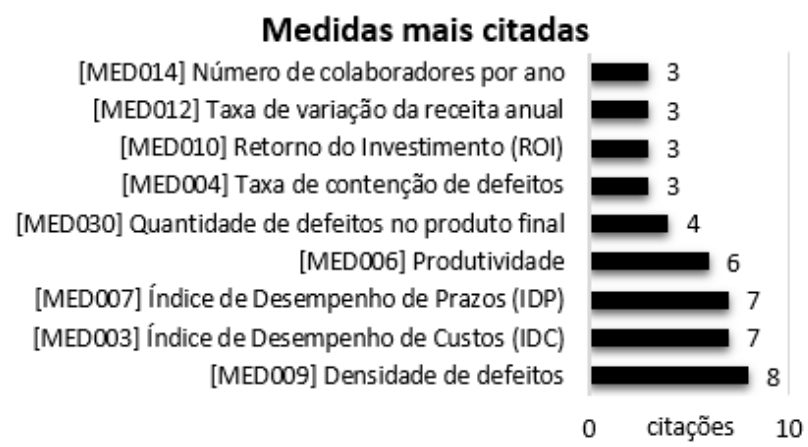

Figura 5 - Medidas mais citadas

Para a questão secundária QS3 - "Em que contexto de melhoria de processos de software os benefícios ocorrem?", foram identificados 29 contextos de ocorrência. Os gráficos da Figura 6 apresentam um panorama geral dos contextos de SPI identificados. No gráfico A é indicada a quantidade benefícios associados ao modelo MR-MPS-SW, no gráfico $\mathrm{B}$ é indicada a quantidade benefícios associados ao modelo CMMI-DEV, no gráfico $\mathrm{C}$ é indicada a quantidade benefícios associados às normas ISO e aos modelos Competisoft e Bootstrap, enquanto o gráfico D apresenta a quantidade benefícios identificados em implementações múltiplas. Os gráficos apontam uma maior concentração dos benefícios identificados nos contextos dos níveis mais baixos do CMMI-DEV (níveis 2 e 3) e do MR-MPS-SW (níveis G, F, E e D).

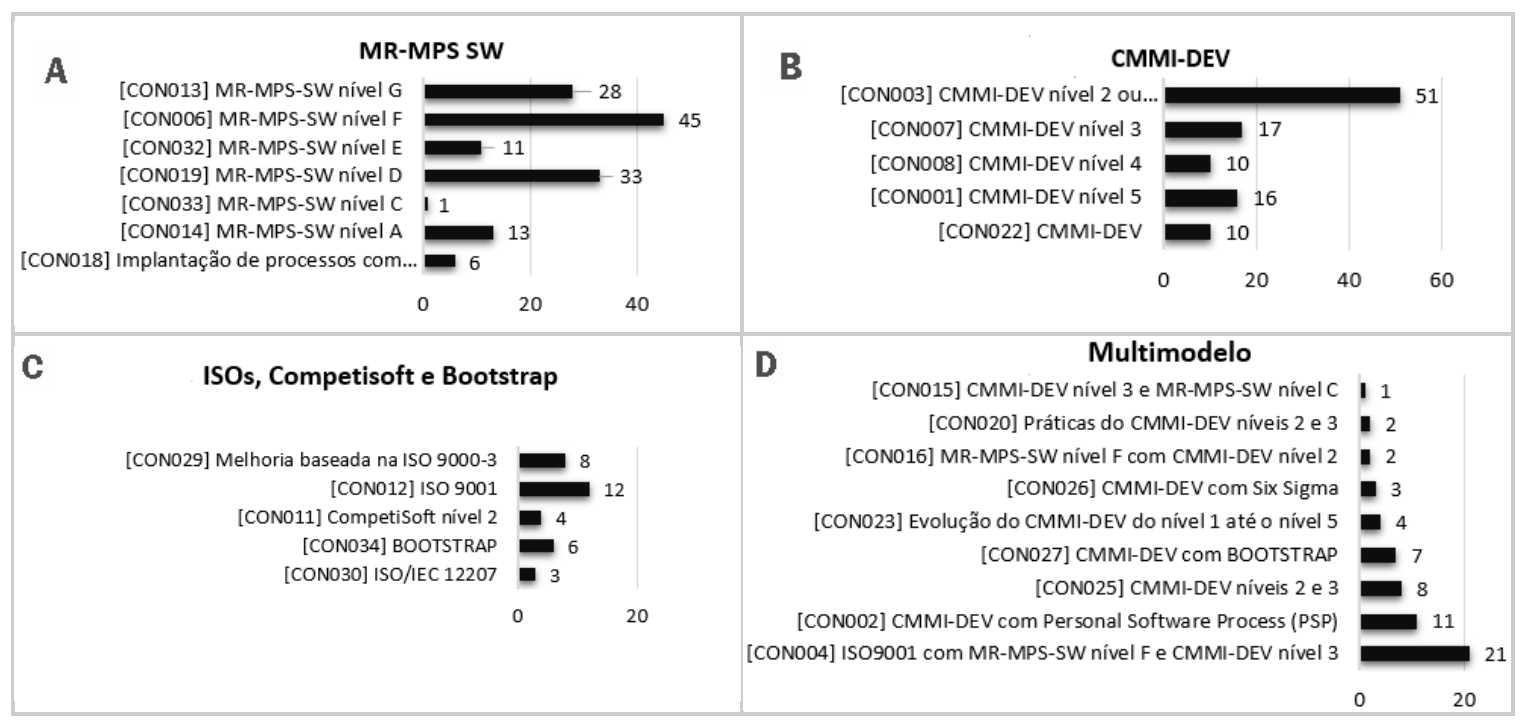

Figura 6 - Quantidade de benefícios associadas a contextos de SPI

Em resposta à questão secundária QS4- "Em que publicações os benefícios foram relatados?", a análise resultou na associação dos benefícios às referências bibliográficas de onde foram extraídos, permitindo rastreabilidade da origem cada benefício. O grafo da Figura 7 apresenta a codificação das referências do benefício [BEN012], onde pode-se observar que o mesmo foi extraído dos artigos [REF022] e [REF002]. 


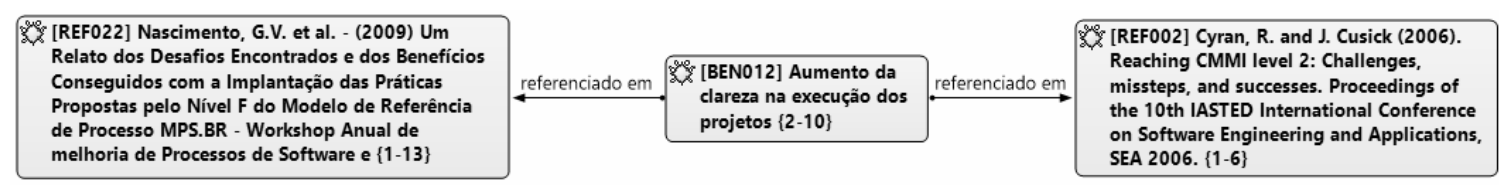

Figura 7 - Exemplo de codificação de referências bibliográficas

Para a questão secundária QS5- “Qual relação entre os benefícios relatados?”, a análise qualitativa realizada nesse estudo permitiu identificar que alguns benefícios podem influenciar na ocorrência de outros, por exemplo, o benefício "aumento da produtividade" pode influenciar positivamente na ocorrência do benefício "aumento do lucro por colaborador". Esta questão é representada no auto relacionamento apresentado na classe de Benefícios do modelo de análise apresentado na Figura 2.

Ao fim do processo de codificação, foi realizada uma auditoria dos procedimentos de codificação em duas etapas. Na primeira etapa, 2 auditores da área de melhoria de processos de software que possuem publicações científicas cuja metodologia inclui procedimentos da Grounded Theory foram selecionados e avaliaram a clareza da descrição dos códigos gerados e a consistência entre o código e os trechos de artigos aos quais está associado. Na segunda etapa, um auditor com larga experiência em projetos de implementação e avaliação em modelos de melhoria de processos de software em organizações avaliou a coerência dos relacionamentos entre os códigos obtidos. As sugestões dos auditores nas etapas de auditoria contribuíram para a padronização e correção de termos e fórmulas, além de garantir que os procedimentos de coleta e análise foram executados de acordo com a metodologia de análise definida.

\subsection{Estrutura do Catálogo de Benefícios}

As informações sobre benefícios capturadas no mapeamento sistemático da literatura foram organizadas em fichas de benefício para compor o catálogo que é composto por: (i) Capa, onde são apresentados os dados de identificação do catálogo (título, autor, filiação, versão e ano de criação); (ii) Seção introdutória, onde o leitor é resumidamente instruído quanto ao conteúdo do catálogo e a metodologia utilizada para coleta dos dados; (iii) Seção de estrutura das fichas, onde é apresentada detalhadamente a estrutura em que as fichas com dados sobre os benefícios estão organizadas; (iv) Sumário, onde são indicadas as páginas de localização das fichas contidas no catálogo; e (v) Capítulos formados pelas categorias de benefícios.

A Figura 8 apresenta a ficha do grupo de benefícios "Aumento da receita/lucro", que agrupa os benefícios "Aumento do lucro por colaborador" e "Aumento do percentual de lucro" e pode gerar "Aumento da satisfação da alta gerência". Está na categoria "Benefícios Relacionados a Ganhos Financeiros e Redução de Custo Operacional”. O catálogo completo encontra-se disponível no apêndice D de [Cruz, 2016]. 


\subsection{Avaliação do artefato}

Para avaliação do artefato produzido nesta pesquisa, as técnicas Grupo Focal [Caplan 1990] e Painel de Especialistas [Pinheiro et al. 2013] foram selecionadas como as mais apropriadas. A desvantagem Grupo Focal em relação ao Painel de Especialistas é necessidade de disponibilidade dos participantes em um mesmo dia e horário, envolvendo questões de deslocamento e disponibilidade de espaço físico. Outra razão que conduziu à seleção da técnica de Painel de Especialistas como mais adequada foi modelo de entrevista individualizado e estruturado, com a possibilidade de mesclar o uso de questionários com discussões. No Grupo Focal, os participantes são deixados livres para discutirem sobre um assunto de interesse da pesquisa sem a condução direta do pesquisador, o que representaria um alto risco para o estudo.

O painel de especialistas atuou tanto como uma técnica para avaliação do catálogo, como uma fonte de novos conceitos e relacionamentos, ressaltando também a característica exploratória desta etapa do estudo. Foram selecionados 4 especialistas em SPI, implementadores e/ou avaliadores em modelos de maturidade, como MR-MPS-SW e CMMI-Dev, com pelo menos 10 anos de experiência em participação em projetos de implementação de SPI, que avaliaram a estrutura e conteúdo do catálogo obtido nesse estudo por meio de um questionário e discussões. Como resultado, foi possível perceber indícios de que as seções contidas nas fichas de benefício são adequadas e relevantes ao contexto organizacional. Todos os especialistas entrevistados concordam que as informações contidas no catálogo podem auxiliar organizações a definirem seus objetivos iniciais de implementação, auxiliar na venda da melhoria de processos de software nas organizações (convencimento da alta gerência em investir em SPI) e ser utilizadas por um gerente para motivar sua equipe a executar práticas de SPI. Por fim, as entrevistas permitiram identificar novos relacionamentos entre os benefícios e pontos de melhoria em descrições de categorias de benefícios e de nomes de benefícios. Foram identificados 37 novos relacionamentos, avaliados por um profissional experiente em SPI com o propósito de mitigar indícios de interpretação inadequada por parte do pesquisador. Como não houve ocorrências de má interpretação, os novos relacionamentos foram incluídos no catálogo.

\subsection{Limitações e Ameaças à validade}

Algumas limitações e ameaças podem ser identificadas nesse estudo. Dentre as limitações, destaca-se o fato de nem todas as fichas de benefícios serem completas. Isso porque, em alguns relatos não foram mencionadas medidas, fórmulas, técnicas ou fatores de influência. Dentre as ameaças à validade identificadas, destacam-se:

Ameaça à validade interna: São eventos não controlados que podem produzir distorções no resultado esperado. Estudos relevantes que usam termos diferentes dos previstos na expressão de busca ou que ainda não haviam sido indexados aos engenhos podem não ter sido selecionados. Apesar da imparcialidade do pesquisador nas entrevistas, não é possível saber se de alguma forma sua presença influenciou nas respostas fornecidas pelos participantes.

Ameaça à validade externa: As ameaças deste tipo prejudicam a generalização dos 
resultados do estudo. A limitação de fontes de dados a relatos de experiência e entrevistas com especialistas limita também a abrangência dos resultados. É possível que novos achados possam ser evidenciados a partir da realização de novas análises e exploração de novas fontes de dados.

Ameaça à validade do constructo: São eventos que podem prejudicar a medição correta no estudo. $\mathrm{O}$ questionário utilizado no painel de especialistas poderia gerar desvio de interpretações, por isso, foi realizada uma rodada piloto para ajustar questões, simplificar e direcionar as respostas. Em alguns momentos é possível que as opções de resposta não tivessem a intensidade da opinião do entrevistado. Além disso, é possível que o valor percebido com relação às opções de resposta varie de acordo com os critérios pessoais de cada participante.

Ameaça à validade de conclusão: Estas ameaças prejudicam o estabelecimento de relacionamentos estatísticos. Neste estudo não foram realizados estudos experimentais, nem testes estatísticos, assim, os resultados não poderão ser considerados conclusivos.

\section{Conclusão}

Este trabalho identificou benefícios reportados por organizações que implementaram SPI baseada em modelos e normas internacionais. Muitas vezes, na decisão por investir em SPI, benefícios são definidos como objetivos iniciais de implementação para que no futuro seja possível avaliar o retorno obtido. Assim, informações sobre experiências obtidas por outras organizações possibilitam que investidores de organizações inexperientes na melhoria de processos de software reflitam com melhor percepção sobre aquilo que se adquiriu ou se deseja adquirir em termos de melhoria de processos.

O estudo incluiu um mapeamento sistemático da literatura, onde foram identificados 57 relatos de experiência de implementação de SPI em que autores relataram benefícios resultantes da adoção de práticas propostas por modelos e normas de SPI. A análise temática realizada sobre esses estudos resultou na catalogação de 121 fichas de benefício com fatores de influência positiva, medidas para acompanhamento, fórmulas de medidas, técnicas para acompanhamento, referências bibliográficas e contextos de ocorrência e cujo índice de completude varia de acordo com a disponibilidade das informações nas fontes analisadas.

Por fim, a estrutura e conteúdo do catálogo de benefícios foram avaliados por especialistas em SPI. Os resultados da avaliação indicaram que as fichas de benefício podem auxiliar organizações a definirem seus objetivos iniciais de implementação e influenciar na motivação, tanto de gestores em decidir por implementar SPI, quanto de colaboradores em executar práticas de melhoria. Também foram obtidos indícios de que os tipos de informação apresentados nas fichas de benefício são adequados e relevantes ao contexto organizacional. Entretanto, novos estudos serão realizados junto à indústria em vista de confirmar os indícios obtidos nesse trabalho.

\section{Agradecimentos}

Os autores agradecem à FAPERJ (projetos E-26/210.643/2016, E-211.174/2016) e à UNIRIO (Edital PQ-UNIRIO no. 01/2016 e 01/2017) pelo apoio financeiro. 


\section{Referências}

Bardin, L. (1977). Análise de conteúdo. Lisboa: Editora Edições 70.

Caplan, S. (1990). "Using focus group methodology for ergonomic design". Ergonomics, v. 33, n.5, p. 527-33.

Cerdeiral, C.T (2008). Uma Abordagem para Gerência e Avaliação de Projetos de Melhoria de Processos de Software do Ponto de Vista da Instituição de Consultoria. Dissertação de M. Sc., COPPE/UFRJ, Rio de Janeiro, Brasil.

CMMI Product Team (2010). CMMI for Development (CMMI-DEV) Version 1.3. Software Engineering Institute. Technical Report CMU/SEI-2010-TR-033. Pittsburgh, PA: Software Engineering Institute, Carnegie Mellon University.

Cruz, D. (2016). "Catálogo de Benefícios Relatados por Organizações que Implementaram Melhoria de Processos de Software.", Dissertação de M. Sc. UNIRIO, Rio de Janeiro, Brasil.

Coleman, G., O'Connor, R. (2008). "Investigating software process in practice: A grounded theory perspective", Journal of Systems and Software, v.81, 5, pp.772-784.

Dresh, A., Lacerda, D.P. E Júnior, J.A.V.A (2015). Design Science Research: Método de Pesquisa para Avanço da Ciência e Tecnologia. Bookman.

ISO/IEC (2003). "ISO/IEC 15504: IT Process Assessment." The International Organization for the Standardization and the International Electrotechnical Commission.

ISO/IEC (2008). ISO/IEC 12207: System and software engineering - Software life cycle processes", The International Organization for the Standardization and the International Electrotechnical Commission.

Mcloughlin, F., e Richardson, I. (2010). "The Rosetta Stone methodology-a benefits driven approach to SPI", In Systems, Software and Services Process Improvement, pp. 201-212, Springer Berlin Heidelberg.

Montoni, M.A. e Rocha, A.R.C. (2011). "Uma Investigação sobre os Fatores Críticos de Sucesso em Iniciativas de Melhoria de Processos de Software". Simpósio Brasileiro de Qualidade de Software (SBQS), Curitiba, PR, Brasil.

Neto, J.B.M, Cardoso, M.P., Bezerra, S. (2013). "RisAgi: Uma Metodologia Ágil para Gestão de Riscos em Projetos de Desenvolvimento de Software", Workshop Anual de Melhoria de Processos de Software e Serviços, Campinas, SP.

Petrersen, K. et al. (2008). "Systematic mapping studies in software engineering", International conference on Evaluation and Assessment in Software Engineering, p. 68-77, Bari, Italy.

Pinheiro J. Q., Farias T. M., Abe-Lima J. Y. (2013). "Painel de especialistas e estratégia multimétodos: reflexões, exemplos, perspectivas", Psico, v. 44, Porto Alegre.

Ramos, C. S., Rocha A. R. E Oliveira, K. M. (2013). "Towards a strategy for analysing benefits of Software Process Improvement”, SEKE, 2013.

Rico D., 2002, Software "Process Improvement (SPI): Modeling Return on Investment (ROI)". Em http://davidfrico.com/dacs02.pdf. Consulta realizada 15/05/2017.

Santos, G. (2011). Influência e Impacto do Programa MPS.BR na Pesquisa Relacionada à Qualidade de Software no Brasil, Simpósio Brasileiro de Qualidade de Software, Curitiba/PR.

SOFTEX (2016), "Melhoria do Processo de Software Brasileiro - Guia Geral MPS de Software", Consulta realizada 15/05/2017.

Solingen, R. V. (2004). "Measuring the ROI of software process improvement.". IEEE Software. V. 21, n.3, pp. 32-38.

Strauss, A., Corbin, J. (2008). Pesquisa Qualitativa - Técnicas e Procedimentos para o desenvolvimento de teoria fundamentada. 2a. Ed., Porto Alegre: Artmed e Bookman.

Travassos, G.H. e Kalinowski, M. (2009). "iMPS 2009: caracterização e variação de desempenho de organizações que adotaram o modelo MPS", SOFTEX, Campinas SP

Unterkalmsteiner, M. et al. (2012). "Evaluation and Measurement of Software Process Improvement-A Systematic Literature Review, Software Engineering”, IEEE, v38, pp398-424 